\section{Avaliação dos padrões de uso de álcool em usuários de serviços de Atenção Primária à Saúde de Juiz de Fora e Rio Pomba (MG)}

\section{Evaluation of the patterns of alcohol consumption at Primary Health Care services in Juiz de Fora and Rio Pomba (MG)}

\section{Resumo}

Uma parte significativa da população brasileira apresenta padrões de consumo de álcool associados a alto risco de desenvolvimento de problemas. Por tal razão, é fundamental avaliá-los na população para traçar estratégias de prevenção primária e secundária ao seu uso. O objetivo deste estudo foi avaliar esse padrão de consumo dos usuários de serviços da Atenção Primária à Saúde das cidades de Juiz de Fora e Rio Pomba, Minas Gerais. Foi aplicado um instrumento de rastreamento - $\mathrm{o}$ AUDIT - a 921 sujeitos recrutados durante as consultas de rotina. Considerando os dados em conjunto, $18,3 \%$ das pessoas avaliadas encontravam-se na faixa de uso de risco, $77,9 \%$ da amostra faziam uso de baixo risco ou eram abstêmios, e 3,8\% pontuaram na faixa sugestiva de dependência. De modo geral, os homens apresentaram comportamento de risco em maior freqüência do que as mulheres, sendo mais prevalente na faixa etária dos 38 aos 47 anos de idade.

Palavras-chave: Cuidados Primários de Saúde. Triagem. Alcoolismo. AUDIT.

Molise de Bem Magnabosco'

Maria Lúcia Oliveira de Souza Formigoni²

Telmo Mota Ronzani'

${ }^{1}$ Departamento de Psicologia da Universidade Federal de Juiz de Fora

${ }^{2}$ Departamento de Psicobiologia da Universidade Federal de São Paulo

Financiamento: Pró-Reitoria de Pesquisa da Universidade Federal de Juiz de Fora (PROPESQ/UFJF) Correspondência: Molise de Bem Magnabosco. Rua B, no 249 - Residencial São José Rondonópolis - MT - CEP: 78730-805. E-mail: molisebm@yahoo.com.br 
Abstract

A significant part of the Brazilian population presents alcohol consumption patterns associated with high risk of development problems. Therefore, it is fundamental to verify the patterns of alcohol consumption among the population in order to outline primary and secondary prevention strategies. The aim of this study is to verify the patterns of alcohol consumption among the users of Primary Health Care in the cities of Juiz de Fora and Rio Pomba, in the state of Minas Gerais. We applied a screening test - the AUDIT - in a sample of 921 people selected during routine consultations. Considering data as a whole, $18.3 \%$ of the evaluated people were at the risk use zone; $77.9 \%$ had low hazardous alcohol use or were abstemious and 3.8\% pointed toward the suggestive dependence zone. In general, men more frequently demonstrated hazardous behavior compared to women, which was also more prevalent in the 38 to 47 year-old-age group.

Keywords: Primary Health Care. Screening. Alcoholism. AUDIT

\section{Introdução}

Estudos apoiados pela Organização Mundial de Saúde constataram que na maior parte dos países em desenvolvimento, entre eles o Brasil, verifica-se, em parcela significativa da população, a ocorrência de padrões de consumo de álcool com elevado grau de risco para diversos problemas de saúde, psicológicos e sociais ${ }^{1-7}$. Tais padrões de consumo se refletem nas taxas de morbi-mortalidade atribuíveis ao uso de álcool que, em 2000, determinaram $3,2 \%$ da mortalidade global. Em 1990, esta estimativa foi de $1,5 \%$. Houve, portanto, um aumento de mais que o dobro no valor encontrado no período de dez anos - indicando, portanto, uma tendência preocupante em termos de saúde pública. No Brasil, o uso abusivo de álcool pode ser responsável por mais de $10 \%$ dos problemas totais de saúde da população ${ }^{1}$.

Em 2001, foi realizado o I Levantamento Domiciliar Sobre o Uso de Drogas Psicotrópicas no Brasil ${ }^{8}$, com amostra representativa da população das 107 cidades do país com número de habitantes superior a 200 mil, que representam $41,3 \%$ da população nacional. Foram incluídas pessoas com idades entre 12 e 65 anos. Observouse que $68,7 \%$ dos entrevistados já haviam feito algum uso de álcool na vida, 5,2\% faziam uso regular de bebidas alcoólicas (bebiam, no mínimo, três a quatro vezes por semana) e $11,2 \%$ preenchiam critérios sugestivos de dependência. Detectou-se diferença entre homens e mulheres quanto ao consumo abusivo de álcool, como ocorre de forma semelhante em outras partes do mundo ${ }^{4,9,10}$. Os homens apresentaram maior freqüência em todos os padrões de consumo. Seu uso na vida foi mais freqüente $(77,3 \%)$ que o das mulheres $(60,6 \%)$ em todas as faixas de idade; bebiam mais regularmente do que elas - cerca de cinco vezes mais na faixa etária acima dos 25 anos. A porcentagem de dependentes entre homens com mais de 18 anos $(23,7 \%)$ foi três vezes maior do que a observada entre mulheres da mesma faixa etária $(7,4 \%)$. 
As mulheres mostraram-se menos vulneráveis à dependência do que os homens: aproximadamente, de cada cinco homens que fizeram uso de álcool na vida, um se tornou dependente; sendo que em relação às mulheres a proporção é bem menor: uma em cada dez ${ }^{8}$.

Para que ações de controle do uso de álcool sejam efetivas, deve-se considerar, em sua elaboração, um contexto mais amplo, no qual estas ações estejam relacionadas às estratégias de saúde pública. Isso advém do fato de que uma abordagem que se concentre apenas nos efeitos biológicos individuais do uso de álcool, desligada do contexto e das crenças que os profissionais possam ter sobre tal uso, torna-se inadequada e/ou pouco efetiva do ponto de vista de sua aplicação à saúde coletiva ${ }^{11}$.

Uma das ferramentas importantes para o planejamento de ações de prevenção em saúde é a utilização de instrumentos de rastreamento, com o objetivo de identificar pessoas que necessitem de níveis diferenciados de intervenção ${ }^{6,11,12}$. Especificamente em relação ao uso de álcool, o rastreamento se justifica pelo fato de que a maioria das pessoas que fazem uso abusivo não é diagnosticada até que tenham se desenvolvido sérias complicações decorrentes do uso de bebidas alcoólicas. A detecção do padrão de consumo pode ser útil para motivá-las a reduzir ou cessar o uso, principalmente se for associada a uma assistência apropriada ${ }^{2,6}$.

Vários estudos ${ }^{13-16}$ discutem a importância da articulação entre a utilização de instrumentos de rastreamento e intervenções adequadas a cada nível de uso, dentre as quais se destacam as intervenções breves (IBs) ${ }^{13}$. Estas, quando dirigidas a usuários de álcool ou outras drogas, podem ser efetivas e menos dispendiosas comparadas a outras modalidades de tratamento, podendo ser aplicadas no contexto da Atenção Primária à Saúde (APS) ${ }^{12-16}$. Tal contexto é propício à realização das intervenções, uma vez que seus profissionais têm maior acesso aos pacientes, facilitando a realização de uma detecção precoce.
No Brasil, até há pouco tempo não existiam estudos a esse respeito, o que constituía uma expressiva lacuna epidemiológica que deve ser preenchida, uma vez que estratégias de controle e prevenção do uso de álcool devem ser propostas com base em dados que indiquem a dimensão do problema existente ${ }^{17}$.

O objetivo do presente trabalho foi avaliar os padrões de uso de álcool entre pacientes que freqüentam serviços de atenção primária à saúde nos municípios de Juiz de Fora e Rio Pomba, ambos situados no Estado de Minas Gerais, a partir da aplicação de um instrumento de rastreamento do uso de álcool feita por profissionais de unidades básicas de saúde.

\section{Metodologia}

\section{Local}

Juiz de Fora e Rio Pomba são municípios brasileiros, situados no Estado de Minas Gerais, na região da Zona da Mata. Juiz de Fora possui aproximadamente $501 \mathrm{mil}$ habitantes. Seus serviços de atenção primária à saúde são compostos de 40 Unidades de Básicas de Saúde (UBS) e 94 Unidades de Saúde da Família, que atendem 49,7\% da população. Rio Pomba, por sua vez, tem população estimada em aproximadamente 17 mil habitantes. Possui 7 estabelecimentos de saúde em nível de atenção básica (5 Unidades Básicas de Saúde e 2 Unidades de Saúde da Família), sendo $50,5 \%$ de sua população cobertos por eles ${ }^{18}$.

\section{Participantes}

O presente estudo é um desdobramento de um projeto multicêntrico para implantação de estratégias de rotinas de rastreamento do uso de álcool e de realização de intervenções breves nos serviços de atenção primária à saúde (APS), apoiado pela Organização Mundial de Saúde (OMS). Juiz de Fora foi uma das cidades brasileiras nas quais ele foi implantado ${ }^{11}$. 
Um instrumento de rastreamento - o AUDIT (Alcohol Use Disorders Identification Test) - foi aplicado a 921 usuários dos serviços de APS dos municípios de Juiz de Fora e Rio Pomba, Minas Gerais, entre os meses de dezembro de 2003 e setembro de 2005. Estes usuários foram recrutados aleatoriamente, segundo critério de conveniência, durante as consultas de rotina por profissionais de saúde que haviam sido submetidos a um treinamento de 16 horas sobre como aplicar o AUDIT na rotina de trabalho e como realizar a intervenção breve para pacientes classificados na zona de risco. Tais profissionais eram médicos $(9,6 \%)$, enfermeiros $(0,7 \%)$, psicólogos (1,3\%), auxiliares de enfermagem (4,3\%) e agentes comunitários de saúde $(27,9 \%)$, bem como estudantes de Psicologia $(39,8 \%)$. No Município de Juiz de Fora, as unidades participantes foram todas as que concordaram em participar do projeto de implantação de rotinas de rastreamento e intervenção breve para o uso de álcool: doze dentre as 134 UBSs de Juiz de Fora, bem como os outros serviços de APS (serviços de saúde da Casa do Servidor da Prefeitura Municipal de Juiz de Fora e da Polícia Militar de Juiz de Fora). Em Rio Pomba, o estudo foi realizado em duas das 7 UBSs, sendo aquelas nas quais trabalhavam estagiárias de Psicologia da Universidade Federal de Juiz de Fora (UFJF).

\section{Instrumento}

$\mathrm{O}$ instrumento utilizado nesta pesquisa foi o AUDIT, que é um dos primeiros testes de rastreamento desenhados especificamente para serviços de APS $^{6}$, sendo recomendado por diversos pesquisadores como alternativa mais eficiente do que inventários como o CAGE e o MAST (Michigan Alcohol Screening Test). Entre suas vantagens está o enfoque nos problemas atuais e não apenas na identificação dos dependentes de álcool, considerando principalmente os usuários dessa substância que apresentam problemas iniciais ${ }^{2,19-21}$.

O AUDIT é composto por 10 questões e avalia o uso recente de álcool, sintomas de dependência e problemas relacionados ao álcool. As respostas a cada questão são pontuadas de 1 a 4, sendo as maiores pontuações indicativas de problemas. Classifica-se o usuário em uma de quatro zonas de risco, de acordo com o escore obtido: zona I (até 7 pontos: indica uso de baixo risco ou abstinência); zona II (de 8 a 15 pontos: indica uso de risco); zona III (de 16 a 19 pontos: sugere uso nocivo) e zona IV (acima de 20 pontos: mostra uma possível dependência). É um instrumento de fácil e rápida aplicação, necessitando apenas de um treinamento simples para a codificação dos dados.

O AUDIT foi validado em vários países, inclusive o Brasil, apresentando bons níveis de sensibilidade $(87,8 \%)$ e especificidade $(81 \%)$ para detecção do uso nocivo de álcool, tendo sido seu desempenho avaliado positivamente em serviços de APS $^{22,23}$. Além disso, na validação brasileira, o AUDIT apresentou uma confiabilidade satisfatória e capacidade de responder às mudanças do consumo ${ }^{22}$.

O AUDIT possibilita uma abordagem atenta para a mudança de foco do problema, não mais no alcoolismo, mas no uso de risco, recaindo a ênfase sobre a detecção precoce de uma maior variedade de problemas relatados pelo uso de álcool. $\mathrm{O}$ rastreamento deixa de ser usado apenas como parâmetro diagnóstico e passa a ser importante também na detecção de fatores de risco - podendo, por fim, conduzir mais à educação para a saúde do que a um tratamento formal ${ }^{21}$.

\section{Análise dos dados}

Para fins de análise, a amostra foi decomposta em grupos, de acordo com a idade, o gênero, padrões de uso de álcool e zonas de risco. As faixas etárias foram definidas a partir dos intervalos quartílicos da amostra, sendo divididas em: 11 a 17 anos, 18 a 27 anos, 28 a 37 anos, 38 a 47 anos e 48 anos ou mais. A freqüência de uso de álcool foi classificada, segundo as 
respostas ao AUDIT, em: nunca; menos que uma vez ao mês; mensalmente; semanalmente; todos ou quase todos os dias. Os serviços de saúde foram divididos em: Unidades Básicas de Saúde (UBS) e outros serviços de APS (serviços de saúde da Casa do Servidor da Prefeitura Municipal de Juiz de Fora e da Polícia Militar de Juiz de Fora).

Visando avaliar a possibilidade de danos físicos, mentais ou sociais decorrentes do uso de álcool, verificou-se, nesta pesquisa, a ocorrência dos seguintes padrões de uso: risco, nocivo e dependência $^{12}$. Classificamos tais padrões estabelecendo uma correspondência entre eles e as zonas de risco, uma vez que tal definição considera não somente a quantidade de doses-padrão de álcool utilizadas, mas a articula com outros comportamentos de risco.

Também foi avaliada na amostra a ocorrência de dias de beber pesado, aqui caracterizada pela ingestão de cinco ou mais doses de álcool em uma única ocasião (questão 3), sendo classificada em: no ano, no mês, na semana, todos ou quase todos os dias.

Uma outra classificação foi estabelecida em função da formação dos aplicadores: nível superior completo (médicos, enfermeiros e psicólogos); nível superior em curso (estagiários de psicologia); nível fundamental ou médio (auxiliares de enfermagem e agentes comunitários de saúde) e auto-aplicação (quando o usuário do serviço respondeu sozinho ao instrumento).

\section{Análise estatística}

Os dados foram digitados e analisados no software estatístico SPSS, versão 15.0. Foram realizadas estatísticas descritivas e inferenciais. Para as variáveis numéricas, foram utilizados testes paramétricos para a comparação entre grupos, quando os pressupostos de distribuição normal e homogeneidade de variâncias foram alcançados. Para a comparação das médias entre duas amostras foi utilizado o Teste $\mathrm{t}$ de Student. Para a comparação das médias entre mais de duas amostras, foi utilizada a Análise de Variância (ANOVA) com posteriores comparações entre os grupos através do teste post hoc de Tukey. Para variáveis categóricas, foi utilizado o teste de Qui Quadrado. Foi estabelecido o nível de significância de $\mathrm{p} \leq 0,05$.

O projeto foi encaminhado e aprovado pelo Comitê de Ética em Pesquisa da Universidade Federal de São Paulo (Unifesp), sendo sua aprovação classificada pelo CEP 0145/03.

\section{Resultados}

A Tabela 1 apresenta os principais dados sociodemográficos da amostra, composta por 921 sujeitos, sendo $94,9 \%$ deles atendidos em Juiz de Fora e 5,1\% em Rio Pomba. A amostra era equilibrada em relação ao gênero (51,9\% homens) e continha representantes de todas as faixas etárias. A maioria dos pacientes $(84,4 \%)$ foi avaliada em UBSs e $15,6 \%$ em outros serviços de APS.

Comparando os serviços, houve uma predominância de homens nos outros serviços de APS (74,3\%), enquanto as UBSs apresentaram uma distribuição equilibrada entre os gêneros (52,3\% de mulheres) $\left(\chi^{2}=34,32 ; \mathrm{p}=0,00\right)$. A idade média (idade

Tabela 1 - Dados sociodemográficos da amostra - valores expressos em porcentagem $(\mathrm{n}=921)$

Table 1 - Sociodemographic data of sample percentage expressed values $(n=921)$

\begin{tabular}{llr}
\hline Gênero & Masculino & 51,9 \\
& Feminino & 48,1 \\
\hline Serviço & Outros serviços de APS & 15,6 \\
& UBS & 84,4 \\
\hline Cidade & Juiz de Fora & 94,9 \\
& Rio Pomba & 5,1 \\
\hline Faixas & $11-17$ anos & 5,2 \\
etárias & $18-27$ anos & 16,0 \\
& $28-37$ anos & 21,2 \\
& $38-47$ anos & 25,6 \\
& 48 anos ou mais & 32,0 \\
\hline
\end{tabular}


em anos \pm DP) dos pacientes avaliados em UBSs $(41 \pm 16)$ foi semelhante a dos pacientes avaliados em outros serviços de APS $(40 \pm 8$ anos $)$

Observamos que a maior parte da amostra total fazia um uso de baixo risco de álcool ou era abstêmia $(77,9 \%)$, sendo que $18,3 \%$ dos sujeitos relataram padrões de consumo que podem ser considerados de risco ou abusivo e apenas 3,8\% níveis sugestivos de dependência.

Como pode ser visto na Tabela 2, os homens se apresentaram dentro de padrões de uso de risco/uso nocivo (respectivamente nas zonas 3 e 4) em freqüência significativamente maior do que as mulheres, o mesmo ocorrendo em relação ao padrão de uso sugestivo de dependência $\left(\chi^{2}=52,16 ; \mathrm{p}<0,001\right)$. A taxa de abstêmios/ usuários ocasionais também foi menor entre os homens $(53,6 \%)$ do que entre as mulheres $(77,9 \%)$. O uso freqüente (semanalmente e todos ou quase todos os dias) foi $19,3 \%$ entre os homens e $7,2 \%$ entre as mulheres $\left(\chi^{2}=67,73 ; p<0,001\right)$. O escore médio do AUDIT foi significativamente $(t=9,19 ; p<0,001)$ mais elevado entre os homens $(6,49$ pontos, $\mathrm{DP}=7,06)$ do que entre as mulheres $(2,90$ pontos, $\mathrm{DP}=4,38)$.

Houve diferenciação quanto ao padrão de consumo de álcool entre as faixas etárias: os sujeitos que tinham entre 38-47 anos apresentaram um comportamento de risco maior que os demais. Houve uma maior concentração dos relatos de uso nocivo de álcool ou de propensão à dependência nessa faixa etária.(5,2\% dos indivíduos com idades entre 38-47 anos foram localizados na zona 3 e $7,0 \%$ na zona 4 quase 2,5 vezes o valor encontrado para 2837 anos). Ainda considerando o uso abusivo, outra grande concentração de indivíduos na zona 3 foi encontrada entre aqueles com 28-37 anos (4,7\%). Quando se avaliou o uso de risco, percebeu-se uma maior incidência dele entre os sujeitos com idades entre $11-27$ anos (23,4\% dos que tinham 11-17 anos e $20,8 \%$ daqueles com 18-27 anos estavam localizados na zona 2). Os indivíduos com 48 anos ou mais, por sua vez, fizeram um uso de álcool mais seguro: em relação ao uso de baixo risco ou abstinência, evidenciou-se uma grande concentração dos sujeitos dessa faixa etária $(85,8 \%$ deles encontravam-se na zona 1$)\left(\chi^{2}=31,83 ; \mathrm{p}=0,00\right)$. Quanto à pontuação total do AUDIT, considerando as diversas faixas etárias, ocorreu diferença entre grupos: aqueles que tinham mais de 48 anos obtiveram uma pontuação me-

Tabela 2 - Zonas de risco de uso de álcool, de acordo com o AUDIT - dados expressos em porcentagem $(\mathrm{n}=921)$

Table 2 - Alcohol use risk zones, according to AUDIT - percentage expressed data $(n=921)$

\begin{tabular}{llcccc}
\hline Zonas de risco & $\begin{array}{c}1 \\
\text { (0-7 pontos) }\end{array}$ & $\begin{array}{c}2 \\
(8-15 \text { pontos })\end{array}$ & $\begin{array}{c}3 \\
\text { (16-19 pontos) }\end{array}$ & $\begin{array}{c}4 \\
\text { (+20pontos) }\end{array}$ \\
\hline Total & & 77,9 & 15,1 & 3,3 & 3,8 \\
\hline Gênero* & Masculino & 69,0 & 19,2 & 5,6 & 6,1 \\
& Feminino & 87,4 & 10,6 & 0,7 & 1,4 \\
\hline Serviço & Outros serviços de APS & 84,7 & 11,1 & 1,4 & 2,8 \\
& UBS & 76,6 & 15,8 & 3,6 & 4,0 \\
\hline Faixa etária** & $11-17$ anos & 74,5 & 23,4 & 2,1 & 0,0 \\
& $18-27$ anos & 75,0 & 20,8 & 1,4 & 2,8 \\
& $28-37$ anos & 75,4 & 16,8 & 4,7 & 3,1 \\
& 38-47 anos & 72,6 & 15,2 & 5,2 & 7,0 \\
& 48 anos ou mais & 85,8 & 10,1 & 2,1 & 2,1 \\
\hline
\end{tabular}

* $\mathrm{p}=0,00$ no teste de Qui-quadrado para a diferença nas zonas de risco do AUDIT.

${ }^{*} p=0.00$ on Chi-square test for the difference at AUDIT risk zones. 
Tabela 3 - Padrões de uso de álcool, de acordo com o gênero e a faixa etária - dados expressos em porcentagem ( $\mathrm{n}=921$ ) Table 3 - Patterns of alcohol use, according to gender and age group - percentage expressed data $(n=921)$

\begin{tabular}{|c|c|c|c|c|c|c|}
\hline \multirow[b]{2}{*}{ Faixa Etária } & & \multicolumn{5}{|c|}{ Padrão de uso de álcool } \\
\hline & & Nunca & $\begin{array}{c}\text { Menos de uma } \\
\text { vez ao mês }\end{array}$ & Mensalmente & Semanalmente & $\begin{array}{c}\text { Todos ou quase } \\
\text { todos os dias }\end{array}$ \\
\hline \multirow[t]{2}{*}{ 11-17 anos * } & HOMENS & 36,4 & 9,1 & 40,9 & 13,6 & 0,0 \\
\hline & MULHERES & 48,0 & 36,0 & 8,0 & 8,0 & 0,0 \\
\hline \multirow[t]{2}{*}{ 18- 27 anos } & HOMENS & 21,0 & 29,0 & 43,5 & 6,5 & 0,0 \\
\hline & MULHERES & 37,8 & 31,7 & 23,2 & 6,1 & 1,2 \\
\hline \multirow[t]{2}{*}{ 28-37 anos * } & HOMENS & 20,4 & 33,7 & 29,6 & 8,2 & 8,2 \\
\hline & MULHERES & 29,0 & 44,1 & 22,6 & 3,2 & 1,1 \\
\hline \multirow[t]{2}{*}{ 38- 47 anos * } & HOMENS & 24,8 & 22,6 & 23,3 & 12,8 & 16,5 \\
\hline & MULHERES & 45,4 & 33,0 & 9,3 & 9,3 & 3,1 \\
\hline \multirow[t]{2}{*}{48 ou mais * } & HOMENS & 35,5 & 25,7 & 21,1 & 10,5 & 7,2 \\
\hline & MULHERES & 56,6 & 28,7 & 8,8 & 4,4 & 1,5 \\
\hline \multirow[t]{2}{*}{ TOTAL * } & HOMENS & 27,2 & 26,4 & 27,2 & 10,7 & 8,6 \\
\hline & MULHERES & 44,0 & 33,9 & 14,9 & 5,6 & 1,6 \\
\hline
\end{tabular}

${ }^{*} \mathrm{p}<0,05$ no teste de Qui-quadrado para os diferentes padrões de uso de álcool entre os gêneros

${ }^{*} p<0.05$ on Chi-square test for the different patterns of alcohol use between genders

nor (média $=3,42 ; \mathrm{DP}=5,10$ ) que os sujeitos que se encontravam nas faixas de $28-37$ anos (média=5,18; DP=5,79; post hoc de Tukey com $\mathrm{p}=0,02$ ) e 38-47 anos (média=6,00; DP=7,75; post hoc de Tukey com $\mathrm{p}=0,00$ ) (ANOVA - $\mathrm{F}=6,26$ ). Quando se comparou o tipo de uso de álcool de acordo com o gênero, considerando-se as diferentes faixas etárias, percebeu-se que os homens apresentaram um padrão de consumo de maior risco que as mulheres em todas as faixas de idade (para maiores informações, ver a Tabela 3).

Dos AUDITs aplicados, 22,3\% o foram por profissionais com ensino superior completo; $39,8 \%$ por aqueles com ensino superior em curso; $32,2 \%$ pelos que tinham ensino fundamental ou médio e $5 \%$ foram resultantes de auto-aplicação. Essa diferença entre as categorias profissionais não apresentou variação em relação ao padrão de consumo dos sujeitos - não houve diferenciação quanto à distribuição dos indivíduos nas várias zonas de risco.

$35,1 \%$ da amostra total relataram a ocorrência de dias de beber pesado. Considerando a freqüência em que ocorrem tais episódios, os serviços apresentaram distribuição semelhante: tanto os usuários das UBSs quanto dos outros serviços de APS relataram uma maior freqüência de dias de beber pesado no ano, com baixa ocorrência em todos ou quase todos os dias. Mais mulheres $(53,1 \%)$ do que homens $(37,3 \%)$ relataram ter ocorrido dias de beber pesado no ano. Estes distribuíram-se também por tal uso no mês $(29,3 \%)$ e na semana $(26,2 \%)\left(X^{2}=7,81 ; p=0,05\right)$. Considerando a idade, a maior parte daqueles que afirmaram a existência de dias de beber pesado com freqüência de todos ou quase todos os dias e na semana concentrou-se nas faixas etárias de 38-47 anos $(11,9 \%$ para a primeira freqüência e $28,6 \%$ para a segunda) e mais de 48 anos ( $8,1 \%$ para a primeira e $27 \%$ para a segunda), sendo que os sujeitos com mais de 48 anos de idade $(51,4 \%)$, juntamente com os que têm entre 11 e 17 anos (50\%), foram os que mais relataram a ocorrência de dias de beber pesado no ano $\left(\chi^{2}=25,01 ; p=0,02\right)$. Não houve diferença entre os gêneros quando se comparou o relato de dias de beber pesado dos indivíduos de diferentes faixas etárias.

Dos 921 sujeitos, 3,8\% apresentaram 
propensão à dependência do álcool, não havendo diferença em tal distribuição entre os serviços. Há mais homens $(6,1 \%)$ do que mulheres $(1,4 \%)\left(X^{2}=13,97 ; p=0,00\right)$ com pelo menos um sintoma de dependência. Em relação às faixas etárias, constatou-se que a propensão à dependência aumenta conforme aumenta a idade (dos sujeitos com 11-17 anos, nenhum apresentou tal propensão, contra $2,8 \%$ dos que tinham entre 18 e 27 anos, 3,1\% daqueles com idades entre 28 e 37 anos e 7,0\% dos que tinham de 38-47 anos) - exceto para aqueles com mais de 48 anos $(2,1 \%)$ $\left(\mathrm{X}^{2}=11,66 ; \mathrm{p}=0,02\right)$.

\section{Discussão}

Nossa amostra apresentou índices de uso regular de álcool semelhantes aos nacionais $^{8}(5,2 \%)$. Contudo, os dados encontrados muitas vezes diferiram da literatura existente - a porcentagem de sujeitos considerados possíveis dependentes encontrada aqui foi $3,8 \%$, enquanto a prevalência de dependentes de álcool no país é $11,2 \%^{8}$. Todavia, essa variação pode se dever mais à diferença de critério para avaliação da dependência do que à diferença percentual de dependentes em si, bem como pode se referir ao critério amostral: no presente estudo definiu-se uma população específica (usuários de UBS) e o outro estudo realizou a coleta de dados em domicílios e na população geral (12 a 65 anos).

Por outro lado, um aspecto que corroborou os dados já existentes diz respeito ao padrão de uso de álcool considerando-se o gênero: na literatura, assim como no presente estudo, os homens apresentaram maior consumo em todos os tipos de uso, fazendo maior uso na vida em todas as faixas de idade e tendo uma maior proporção de dependentes do que as mulheres ${ }^{8}$. Considerando dados de outros países ${ }^{11}$ quanto ao uso de risco/nocivo de álcool em APS, uma menor porcentagem tanto de mulheres quanto de homens desta amostra fez tal uso: $11,3 \%$ das mulheres deste estudo e $40 \%$ em outros países; $24,8 \%$ dos homens deste estudo e $50 \%$ em outros países.

Nos Estados Unidos, $10 \%$ a $36 \%$ dos usuários da APS fazem uso abusivo de álcool ou são dependentes ${ }^{24}$. Considerando o abuso como qualquer uso de risco ${ }^{12}$ sendo, pois, caracterizado pelas zonas 2, 3 e $4-, 22,2 \%$ dos sujeitos do presente estudo fazem uso abusivo de álcool. Os homens apresentaram padrão de uso abusivo maior do que as mulheres - 30,9\% contra $12,7 \%$.

As comparações feitas até o momento entre os resultados encontrados neste estudo e os dados já existentes podem muitas vezes não ser precisas, haja vista a diferença entre as populações estudadas, assim como entre os critérios utilizados para definição dos fatores e os instrumentos. Apesar disso, é válido estabelecer tais comparações, uma vez que elas nos indicam tendências de comportamentos.

Quando comparamos os serviços, percebemos que, apesar de os outros serviços de APS terem prevalência de indivíduos do gênero masculino e uma maior concentração de sujeitos nas faixas etárias de maior risco (28-37 e 38-47 anos), e apesar de as UBSs apresentarem mais sujeitos na faixa etária de menor risco (mais de 48 anos), não houve diferenciação quanto ao padrão de consumo de álcool (seja ele zona, dias de beber pesado ou dependência).

Considerando as diferentes zonas de risco, o padrão de consumo aumentou conforme aumentou a idade (daqueles que se encontram nas zonas 3 e 4 , a menor parte deles tem 11-17 e 18-27 anos, seguidos por aqueles com 28-37 anos, tendo a maior porcentagem deles 38-47 anos), sendo tal tendência modificada na faixa acima de 48 anos, que apresentou menor uso de risco - redução essa também demonstrada em outros estudos ${ }^{25-27}$.

Há, no Brasil, proibição legal de qualquer uso ou comercialização de bebidas alcoólicas aos considerados menores de idade. No presente estudo, os sujeitos com idades entre 11 e 17 anos relataram um consumo de álcool de baixo risco, consi- 
derando todas as variáveis (dias de beber pesado, zona de risco e dependência), não tendo sido encontrado nenhum sujeito desta faixa etária com propensão à dependência.

Apesar de não ter havido diferença estatística entre os serviços, um aspecto que chamou atenção foi a alta prevalência de dias de beber pesado no mês $(34,7 \%$ nos outros serviços de APS e $26,6 \%$ nas UBSs) e na semana $(20,4 \%$ nos outros serviços de APS e $24,8 \%$ nas UBSs). Tendo em vista que, nesse tipo de uso, há ingestão de elevadas quantidades de álcool em uma única ocasião, a alta freqüência dele pode gerar vários problemas de saúde, bem como danos sociais e legais ${ }^{1-6}$.

A variação do consumo de álcool encontrada entre os diferentes serviços pode ter ocorrido em função de, nos outros serviços de APS, haver uma relação hierárquica forte, além de um vínculo empregatício - subordinação essa que pode gerar um receio de punição por parte dos funcionários, mesmo sendo afirmada a confidencialidade dos dados. Tal fato pode ter acarretado problemas referentes ao relato de uso de álcool por parte dos empregados, contribuindo para uma minimização desse relato. Esses dados podem, pois, apontar uma limitação da aplicabilidade do AUDIT enquanto instrumento de triagem de auto-relato em situações de trabalho.

\section{Considerações finais}

O presente estudo é relevante, pois não há dados nacionais sobre o consumo de álcool na população usuária da APS. Além disso, é a partir da averiguação dos padrões de uso de álcool dos diferentes grupos que se torna possível elaborar estratégias e políticas públicas de controle e prevenção de tal uso para grupos específicos.

Demais estudos nesse sentido poderiam realizar uma amostragem sistematizada da população, estabelecendo-se uma estratificação da amostra. Além disso, poderiam ser consideradas outras variáveis que complementariam as análises, como renda familiar ou classe social e escolaridade.

As limitações do presente artigo foram: não foi realizada uma sistematização do grupo amostral (a amostra foi feita segundo critério de conveniência); os dias e horários de aplicação do inventário nos serviços de saúde foram escolhidos aleatoriamente; o número de AUDITs aplicados nos outros serviços de APS e em Rio Pomba foi muito pequeno quando comparado ao de aplicações tanto em UBSs quanto em Juiz de Fora - fator esse que pode enviesar os dados. De qualquer forma, as diferenças apresentadas entre os serviços sugerem uma tendência de variação entre as comparações realizadas.

Pesquisas como essa mostram a importância de se considerar, no contexto da saúde pública, não só a dependência do álcool como problema, mas também o uso de risco. Isso se justifica pelo fato de que tal uso traz sérios problemas à população, que podem ser de ordem física, psicológica/emocional ou social. Dessa forma, ressalta-se a necessidade da prevenção e da educação para a saúde, com a conscientização da população a respeito das conseqüências que o uso abusivo de álcool pode acarretar - medida essa que pode ser realizada na APS através de suas equipes de saúde.

\section{Agradecimentos}

Este estudo foi desenvolvido como parte de um projeto multicêntrico internacional, coordenado por Thomas F. Babor e Jonh C. Higgins-Biddle (Department of Community Medicine and Health Care, University of Connecticut Health Center, Farmington, Connecticut), Erikson Felipe Furtado, (Departamento de Psiquiatria da FMUSP-RP), Isisdore Obot (Department of Mental Health and Substance Dependence - World Health Organization). Agradecemos o apoio financeiro da Pró-Reitoria de Pesquisa da Universidade Federal de Juiz de Fora (PROPESQ/UFJF), às Secretarias Municipais de Saúde dos municípios de 
Juiz de Fora e Rio Pomba, à Organização Mundial da Saúde e aos profissionais de saúde das unidades onde foram coletados os dados. Agradecemos, ainda, a colaboração dos alunos da UFJF: Michaela Bitarello do Amaral, Priscila Montianéle de Castro, Marcela Monteiro de Oliveira, Cristiane de Mesquita Silva, Andréia Fernandes Teixeira, Melissa Fioravante, Tatiana de Castro Amato, Ameli Gabriele B. Fernandes, Nelimar Ribeiro de Castro, Gabriela Guerra e Sílvia Gomes, que participaram da fase de coleta de dados. Somos gratos também ao Prof. Dr. José Carlos Fernandes Galduróz, do Departamento de
Psicobiologia da Universidade Federal de São Paulo, pelas sugestões e revisão do artigo.

\section{Colaboradores}

M. B. Magnabosco participou do planejamento da pesquisa, da coleta e análise dos dados e da redação do artigo. M. L. O. S. Formigoni participou do planejamento da pesquisa, da supervisão da análise dos dados e da redação do artigo. T. M. Ronzani participou do planejamento, análise dos dados e redação do artigo e coordenou o projeto no município de Juiz de Fora.

\section{Referências}

1. Meloni, JN, Laranjeira R. Custo social e de saúde do consumo do álcool. Rev Bras Psiquiatr 2004; 26(I Suppl); 7-10.

2. Bush K, Kivlahan DR, MacDonell MB, Fihn SD, Bradley KA. The AUDIT alcohol consumption questions. Arch Intern Med 1998; 158: 1789-95.

3. Health Risks and Benefits of Alcohol Consumption. Alcohol Res Health 2000; 24(1): 5-11.

4. World Health Organization. Global Status Report on Alcohol. Geneva: World Health Organization; 2001.

5. Room R, Babor T, Rehm J. Alcohol and Public Health. Lancet 2005; 365: 519-30.

6. Babor TF, Higgins-Biddle JC, Saunders JB, Monteiro MG. AUDIT: teste para identificação de problemas de álcool roteiro para uso em atenção primária. Ribeirão Preto: PAI-PAD, 2003.

7. Duarte PCAV, Carlini-Cotrim B. Álcool e violência: estudo dos processos de homicídio julgados nos Tribunais do Júri de Curitiba, PR, entre 1995 e 1998. J Bras Dep Quím 2000; 1(1): 17-25.

8. Carlini EA, Galduróz JCF, Noto AR, Nappo SA. I Levantamento Domiciliar Sobre o Uso de Drogas Psicotrópicas no Brasil. São Paulo: CEBRID - UNIFESP, 2002.

9. Royo-Bornada MA, Cid-Ruzafa J, Martin-Moreno JM, Guallar E. Drug and alcohol use in Spain: consumption habits, attitudes and opinions. Public Health 1997; 111, $277-84$.

10. López-Marina V, Romero GP, García RA, Fernández PB, Montané EG, Jordana NM. Evaluación del cribado y la efectividad de una intervención breve en bebedores de riesgo atendidos en consultas de atención primaria. Aten Primaria 2005, 36(5):261-8.
11. Ronzani TM, Ribeiro MS, Amaral MB, Formigoni MLOS. Implantação de rotinas de rastreamento do uso de risco de álcool e de uma intervenção breve na atenção primária à saúde: dificuldades a serem superadas. Cad Saúde Publ2005, 21(3): 852-61.

12. Babor T, Higgins-Biddle JC. Intervenções Breves: para o uso de risco e nocivo de álcool - manual para uso em atenção primária. Ribeirão Preto: PAI-PAD, 2003.

13. Marques ACPR, Furtado EF. Intervenções breves para problemas relacionados ao álcool. Rev Bras Psiquiatr 2004; 26 (I Suppl): 28-32.

14. Ribeiro M. Organização dos serviços para o tratamento de dependência do álcool. Rev Bras Psiquiatr 2004; 26 (I Suppl): 59-62.

15. Samet JH, Rollnick S, Barnes H. Beyound CAGE: a brief clinical approach after detection of substance abuse - a set of four questions to measures substance abuse. Arch. Intern Med 1996; 156: 2287-93.

16. Friedmann P, MdCullough D, Chin M, Saitz R. Screening and Intervention for Alcohol Problems: a national survey of primary care physicians and psychiatrists. J Gen Intern Med 2000; 15: 84-91.

17. Henrique IFS, De Micheli D, Boerngen de Lacerda R, Lacerda LA, Formigoni MLOS. Validação da versão brasileira do teste de triagem do envolvimento com álcool, cigarro e outras substâncias (ASSIST). Rev Assoc Med Bras 2004; 50 (2):199-206.

18. Banco de dados do Sistema Único de Saúde. Cadernos de Informações de Saúde. http://tabnet.datasus.gov.br/ tabdata/cadernos/mg.htm (acessado em 30de março de 2006). 
19. Daeppen JB, Yersin B, Landry U, Pécoud A, Decrey H. Reliability and validity of the Alcohol Use Disorders Identification Test (AUDIT) imbedded within a General Health Risk Screening Questionnaire: results of a survey in 332 primary care patients. Alcohol Clin Exp Res 2000; 24 (5): 659-65.

20. Fleming MF. Screening and brief intervention for alcohol disorders. Fam Pract 1993; 37(3): 231-4.

21. Allen JP, Litten RZ, Fertig JB, Babor T. A review of research on the Alcohol Use Disorders Identification Test (AUDIT). Alcohol Clin Exp Res 1997; 21 (4): 613-18.

22. Mendéz EB. Uma Versão Brasileira do AUDIT (Alcohol Use Disorders Identification Test) [dissertação de mestrado]. Pelotas: Universidade Federal de Pelotas; 1999.

23. Maciel CD. Diagnóstico de Dependência de Álcool entre os Pacientes Internados em enfermarias psiquiátricas do Instituto Raul Soares e Hospital Galba Velloso: há subdiagnóstico? [monografia de especialização]. Belo Horizonte: Instituto Raul Soares - FHEMIG, 1999.
24. Bradley KA, Bush KR, McDonell MB, Malone T, Fihn SD. Screening for problem drinking - comparison of CAGE and AUDIT. J Gen Intern Med 1998; 13:379-88.

25. York JL, Welte J, Hirsch J. Regulation of alcohol intake with advancing age. Alcohol 2005; 36(1):41-6.

26. Molgaard CA, Nakamura CM, Stanford EP, Peddecord KM, Morton DJ. Prevalence of alcohol consumption among older persons. J Community Health 1990; 15(4):239-51.

27. Saunders PA, Copeland JRM, Dewey ME, Davidson IA, McWilliam C, Sharma VK, et al. Alcohol use and abuse in the elderly: Findings from the Liverpool longitudinal study of continuing health in the community. Int J Geriatr Psychiatry 2004; 4: 103-8.

Recebido em: 05/07/06 Versão final reapresentada em: 27/07/07 Aprovado em: 13/08/07 